with a new diagnosis of biopsy-proven GCA in 1986-2007 were retrospectively identified. Patients were followed from GCA diagnosis to death, migration or September 2011. Comparisons were performed using Chi-square and rank sum tests, Kaplan-Meier methods and Cox models.

Results: The study included 110 patients in the Olmsted and 144 in the Reggio cohort. Compared with the Olmsted cohort, patients from the Reggio cohort were younger (mean $\pm S D$ age $74.6 \pm 7.4$ years vs $77.8 \pm 7.6, p=0.002$ ), more likely to have cranial symptoms $(93 \%$ vs $86 \%, p=0.048)$, temporal artery abnormalities at physical examination $(68 \%$ vs $42 \%, p<0.001)$, partial or complete unilateral or bilateral permanent vision loss ( $21 \%$ vs $6 \%, \mathrm{p}=0.001)$, systemic symptoms $(67 \%$ vs $46 \%, p=0.001)$ and polymyalgia rheumatica at or before GCA diagnosis $(47 \%$ vs $26 \%, p<0.001)$. Scalp tenderness was less common in the Reggio cohort ( $36 \%$ vs $49 \%, p=0.033$ ). ESR and CRP were higher (mean $88 \pm 29 \mathrm{~mm} / \mathrm{h}$ vs $73 \pm 77, p<0.001$ and mean $89.0 \pm 60.2 \mathrm{mg} / \mathrm{L}$ vs $35.2 \pm 43.4, p<0.001$ respectively) and hemoglobin lower (mean $11.2 \pm 1.4 \mathrm{~g} / \mathrm{dl}$ vs $11.8 \pm 1.4, \mathrm{p}=0.004$ ) in Reggio than in the Olmsted cohort. Patients from the Olmsted cohort received a higher initial prednisone dose (mean $53.6 \pm 15.3 \mathrm{mg} /$ day vs $49.5 \pm 12.8, \mathrm{p}=0.001$ ). There were no differences in relapse rates, cumulative glucocorticoid (GC) dosages at 1,2 and 5 years, and time to first GC discontinuation. However, the Reggio cohort reached a prednisone dose $<10 \mathrm{mg} /$ day sooner (median 4.9 months vs 7.9 , $\mathrm{p}=0.012$ ) and had a first relapse later (median 13.6 months vs $7.9, \mathrm{p}=0.003$ ) than the Olmsted cohort. Patients from the Reggio cohort had a significantly higher mortality compared to those from the Olmsted cohort (HR 1.72, 95\% Cl 1.12-2.65 adjusted for age and sex).

Conclusions: Genetic and/or environmental factors may contribute to the differences in clinical characteristics and disease outcomes observed in this study comparing patients with GCA from North America and Southern Europe.

Disclosure of Interest: None declared

DOI: 10.1136/annrheumdis-2017-eular.5156

\section{FRI0324 SMALL VESSEL VASCULITIS SURROUNDING A PRESERVED TEMPORAL ARTERY: A DIAGNOSTIC ALGORITHM TO ASSESS CLINICAL SIGNIFICANCE}

G. Espigol Frigole ${ }^{1}, M$. Terenas $^{2}$, S. Prieto-Gonzalez ${ }^{1}$, R. Alba ${ }^{3}$,

J. Hernandez-Rodriguez ${ }^{1}$, E. Planas-Rigol ${ }^{3}$, M. Corbera-Bellalta ${ }^{3}$,

N. Terrades ${ }^{3}$, M.C. Cid ${ }^{1} .{ }^{i}$ Systemic and Autoimmune Diseases Department, Hospital Clinic of Barcelona, Barcelona, Spain; ${ }^{2}$ Medicine Department, Hospital de Santa Maria, Lisboa, Portugal: 3 Institut d'Investigacions Biomèdiques Pi i Sunyer (IDIBAPS). CELLEX, Barcelona, Spain

Background: Systemic vasculitides are complex and heterogeneous diseases with overlapping features that frequently pose a diagnostic challenge to clinicians. The temporal artery biopsy (TAB) is the gold standard for the diagnosis of giant cell arteritis (GCA) but, occasionally, TAB show inflammation of small vessels surrounding a spared temporal artery (SVV) as the only pathologic finding. Ultimate diagnosis and, consequently, optimal treatment remain uncertain in these patient. Objectives: To analyze the final diagnosis of patients with SVV surrounding a spared temporal artery after a pre-established diagnostic algorithm and to identify clinical and laboratory findings with potential usefulness in predicting the ultimate diagnosis.

Methods: Patients with TAB showing SVV were subjected to the diagnostic algorithm displayed in figure 1, completed by at least 1 year follow-up. Clinical and laboratory features at the time of diagnosis were recorded. The algorithm led to the following final diagnosis: GCA, other systemic vasculitis and undetermined condition. Chi-squared and ANOVA tests were used for statistical comparison using IBM SPSS Statistics 20.

Results: From 1998 to 2007, 380 TAB were performed in our institution. Biopsy disclosing small vessel inflammation surrounding a normal temporal artery (SVV) was described in $47(12 \%)$ patients. In all patients TAB was selected as the first invasive procedure because GCA was initially considered the most likely diagnosis. Accordingly, $24(51 \%)$ fulfilled at least 3 ACR classification criteria for GCA. 7 patients declined to undergo subsequent work-up to complete the diagnostic algorithm, 10 died or were lost to follow -up before completing 1 year. All of them were excluded. The study cohort consisted of 30 patients (19 women and 11 men) aged $77 \pm 10.4$ years followed for $55.16 \pm 55.20$ months. In 13 patients the final diagnosis was consistent with GCA based on the absence of SVV in other territories, large-vessel inflammation by imaging or subsequent development of aortic aneurysm; in $12 \mathrm{SVV}$ was subsequently demonstrated in other territories and were diagnosed with other systemic vasculitis (7 AAV, 0 cryo, 3 PAN, 0 vasculitis associated to autoimmune diseases, 2 unclassified small vessel vasculitis), and in 5, diagnosis remained undetermined. No significant differences in clinical or routine laboratory abnormalities were found among patient subgroups stratified according to the final diagnosis.

Conclusions: Inflammation of small vessels surrounding a spared temporal artery in a TAB conveys a substantial diagnostic uncertainly. After a detailed diagnostic work-up most of patients can be diagnosed with GCA. However other systemic vasculitis requiring more aggressive treatment may disclose similar histopathologic findings and are also frequent and diagnosis remains uncertain in a substantial proportion of cases. Search for more accurate molecular biomarkers is necessary for a better interpretation of these findings

Acknowledgements: "Supported by PI15/00092, Plan Estatal de Investigación Científica y Técnica de Innovación 2013-2016 y cofinanciado por el ISCIII-
Small vessel vasculitis surrounding a normal temporal artery diagnostic algorithm

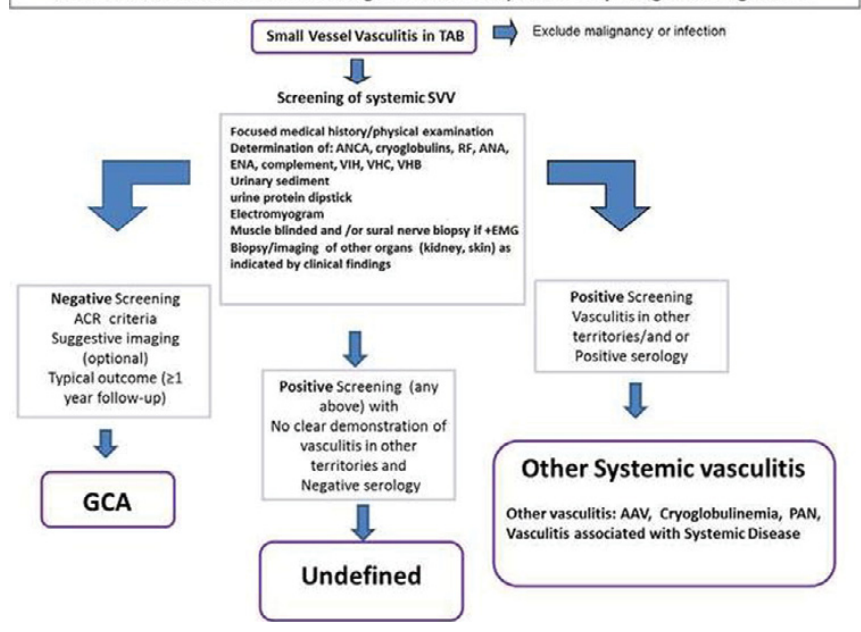

Figure 1

Subdirección General de Evaluación y el Fondo Europeo de Desarrollo Regional (FEDER)"

Disclosure of Interest: None declared

DOI: 10.1136/annrheumdis-2017-eular.5504

\section{FRI0325 PREVALENCE OF TAKAYASU ARTERITIS IN YOUNG WOMEN WITH ACUTE ISCHEMIC HEART DISEASE}

G. Cavalli, A. Tomelleri, E. Baldissera, L. Dagna. Unit of Immunology, Rheumatology, Allergy and Rare Diseases (UniRAR), Vita-Salute San Raffaele University, Milan, Italy

Background: Takayasu arteritis (TA), a systemic vasculitis typically occurring in female patients aged $\leq 40$, can affect the coronary arteries and cause ischemic heart disease. The prevalence of TA among young females with acute ischemic heart disease is undetermined.

Objectives: In this study, we investigated the prevalence of TA in young women presenting with ischemic heart disease in the Emergency Department.

Methods: We conducted a retrospective evaluation of the hospital records of 172,790 consecutive female patients aged $<45$, who accessed the Emergency Department of our institution over 8 consecutive years (2007-2015). The prevalence of TA and of other etiologies of ischemic heart disease was determined. Diagnosis of TA was established based on the 1990 American College of Rheumatology criteria.

Results: Overall, 2,090 women aged $<45$ presented to the Emergency Department with chest pain, dyspnea, palpitations, angina, heart failure, or cardiac arrest; 40 had confirmed acute ischemic heart disease. The etiology was "classic" atherosclerosis in 24 cases $(60 \%)$, TA in 4 cases $(10 \%)$, vasospasm and sympathomimetic drug abuse in 3 cases each (7.5\%), coronary artery dissection and microvascular angina in 2 cases each $(5 \%)$, Takotsubo and radiation-induced cardiomyopathy in 1 case each $(2.5 \%)$.

Conclusions: Although a diagnosis of TA is likely to be overlooked, TA is not infrequent in younger females presenting with acute ischemic heart disease. Specifically, TA accounted for $10 \%$ of cases of acute ischemic heart disease in female patients aged $<45$, a finding relevant to the diagnosis and management of these young patients.

Disclosure of Interest: None declared

DOI: 10.1136/annrheumdis-2017-eular.2298

\section{FRI0326 RECOMMENDATIONS FOR THE MANAGEMENT OF NEURO-BEHÇET DISEASE BY JAPANESE RESEARCH COMMITTEE FOR BEHÇET DISEASE}

H. Kikuchi ${ }^{1}$, T. Sawada ${ }^{2}$, M. Okada ${ }^{3}$, M. Takeno ${ }^{4}$, M. Kuwana ${ }^{4}$

Y. Ishigatsubo ${ }^{5}$, I. Kawachi ${ }^{6}$, H. Mochizuki $^{7}$, S. Kusunoki ${ }^{8}$, S. Hirohata ${ }^{9}$.

${ }^{1}$ Department of Internal Medicine, Teikyo University School of Medicine;

${ }^{2}$ Department of Internal Medicine 3, Tokyo Medical University School of Medicine; ${ }^{3}$ Immuno-Rheumatology Center, St. Luke's International Hospital; ${ }^{4}$ Department of Allergy and Rheumatology, Nippon Medical School Graduate School of Medicine, Tokyo; ${ }^{5}$ Department of Internal Medicine and Clinical Immunology, Yokohama City University Graduate School of Medicine, Yokohama, Japan, Kanagawa; ${ }^{6}$ Department of Neurology, Niigata University Medical and Dental Hospital, Niigata; ${ }^{7}$ Department of Neurology, Osaka University Graduate School of Medicine; ${ }^{8}$ Department of Neurology, Kinki University School of Medicine, Osaka; ${ }^{9}$ Department of Rheumatology and Infectious Diseases, Kitasato University School of Medicine, Kanagawa, Japan

Background: Central nervous system involvement is one of the most serious complications in Behçet's disease (BD). This condition is referred to as neuro- 